\title{
Naturaleza de la sentencia de segunda instancia
}

\author{
Eduardo J. Couture
}

Professor de la Facultad de Derecho y Ciencias Sociales de la Universidad de Montevideo.

\section{Planteo}

¿Cual es la naturaleza jurídica del fallo de segunda instancia? - ¿Constituye una unidad con el de primera instancia o, por el contrario, es independiente de aquél? Cuando confirma, ¿su contenido es declarativo o constitutivo? Cuando revoca, ¿sus efectos son autónomos o se hallan ligados al fallo de primera instancia?

La doctrina acostumbra a examinar estos problemas como una prolongación de otro que, tradicionalmente, se denomina "naturaleza jurídica de la sentencia sometida a impugnación". Sobre este último existe tal cantidad de trabajos que todo resúmen es aquí imposible.

Sin perjuicio de ir refiriendo las mas importantes de esas contribuciones a lo largo de este estudio, nos proponemos examinar la naturaleza del fallo de segunda instancia frente a un caso particular. Nos servimos así de un ejemplo, para llegar a consideraciones generales de doctrina. Vale decir, la inversa del camino habitual.

La delicadeza del caso nos impone esta inversión.

Utilicemos el siguiente ejemplo: 
Un marido, inmediatamente después de dictada la sentencia de divorcio en su favor, adquiere por compraventa un bien raiz. Apelada esa sentencia por el cónyuge, se resuelve la segunda instancia por sentencia confirmatoria de la primera instancia. Se trata de saber si el bien adquirido pertenece exclusivamente al marido o si pertenece a la sociedad conyugal.

Si la sentencia de segunda instancia surte efectos para el futuro y no tiene retroctividad alguna, el bien adquirido es de carácter ganancial y debe dividir-se entre marido y mujer. Si, por el contrario, la sentencia de segunda instancia, confirmatoria de la de primera, retrotrae sus efectos a la fecha en que fué dictado el fallo de primera instancia, el bien adquirido es propio del marido y no debe compartirlo con su esposa.

El punto es de extrema dificultad y ha sido considerado muy diversamente por la doctrina. La incertidumbre que depara es una consecuencia natural de esa dificultad.

Tratemos, pues, de examinarlo con la mayor lucidez posible.

\section{Breve panorama de doctrina.}

La naturaleza del fallo de primera instancia pendiente de apelación ha preocupado reiteradamente a la doctrina. Conviene tener en cuenta quanto se ha dicho sobre este particular, porque constituye un antecedente necesario para nuestra investigación.

La sentencia de primer grado, dice Pavanini (1), ha sido considerada como una decisión sometida a condición

(1) Pavanini, Contributto allo studio del giudizio civile di rinvio, Padova, 1937, p. 33. Análogas esquematizaciones pueden hallarse en los estudios de Cоцомво, Naturaleza juridica dela sentencia sometida a recurso en Rev. D. Pr., 1947, I, p. 488 y FaIren Guillen, Doctrina general de los medios de impugnación y parte general del derecho procesal, en Rev. D. Pr. esp., 1949, p. 297. 
resolutoria (2), o a condición suspensiva (3), o a revocación (4); sea como un elemento que con el concurso de: otro elemento (el transcurso del término) llegará a ser una declaración del derecho (5), - sea finalmente y en términos más generales, como una providencia completa $y$ eficaz pero no definitiva, que podrá llegar a ser destruída por la eventualidad de la nueva sentencia (6).

Este complicado panorama de doctrina requiere alguna ordenación.

El autor citado lo resume así:

"Las varias tesis relativas a la condición de la sentencia - sujeta a impugnación, pueden reducirse sustancialmente a dos grandes categorías; una, considera la sentencia sometida a impugnación como una situación jurídica. que llegará a ser la sentencia de la causa sólo cuando, con. el transcurso de ciertos términos, resulte imposible un pronunciamiento de segundo grado; otra, sostiene que la sentencia del juez se encuentra desde el principio provista de: normal eficacia imperativa, salvo que sea declarada ine-xistente cuando una nueva sentencia de apelación modi-. fique total o parcialmente los efectos" (7).

(2) Korrer, Prozess als Rechtsverhältnis, ps. 71 y 72; el mismo, Zivilprozessrecht, p. 354; MoRTarA, Commentario, t. 4, p. 117: CaSati, nota de jurisprudencia en Rivista di diritto civile, 1912, p. 866 .

(3) VASSAlli, La sentenza condizionale, n. 31; CAlAMANdrei, La cassazione civile t. 2, p. 198; BETTI, Diritto processuale civile. p. 644 .

(4) Rocco (U), L'autoritá della cosa giudicata, p. 252.

(5) Chiovenda, Principii, p. 925; el mismo, Istituzioni, t. 2 , p. 105; Menestrina, I lpassaggio in giudicato della sentenza dí primo gado en Riv. D. P., 1928, II, p. 211; CRistofollini, Efficacio: della sentenza nel tempo, en la misma revista, 1935, I, p. 302; CASTElET, Natura giuridica della sentenza soggetta a gravame, en Foro Italiano, 1933, I, p. 609.

(6) Carnelutti, Lezioni t. 4, n. 3:11; Biond, I poteri dell giudice di inibitoria, publ. en Studi Sassaresi, p. 21 del extracto.

(7) Pavanini op. cit., p. 33. 
Tratemos de ver lo que existe de exacto en estas dos proposiciones.

\section{Una distinción necesaria.}

Conviene comenzar haciendo una distinción que evite: tratar como si fuera uno solo, dos problemas diferentes.

Esta distinción consiste en considerar, por un lado, el. caso de la sentencia de primera instancia durante el plazo. (en nuestro derecho, de cinco días) dentro del cual puede. interponerse el recurso de apelación. Por otro lado debe examinar-se, como asunto diferente, la sentencia de primera instancia cuando ha sido deducido contra ella el recurso de apelación y se halla pendiente de fallo la segunda instancia.

Son dos situaciones distintas, de consecuencias también distintas. Conviene añadir que este problema tiene una gran significación en legislaciones que, como la francesa y la italiana, tienen el procedimiento de ejecución provisoria de la sentencia. Esta circunstancia obliga a los autores a considerar el tema acudiendo a cada instante a esta institución. Diferente es la situación en derechos como el nuestro que no tienen ejecución provisional de la sentencia de primera instancia.

Tratemos, pues, de examinar muy brevemente el caso especial de la sentencia durante el término en que puede ser apelada, ya que es ésa una situación relativamente fácil, que conviene descartar de antemano, para evitar que interfiera en el exámen de la sentencia de segunda instancia.

Durante el término de cinco días dado para apelar, la sentencia es un acto jurídico cuya eficacia depende de la actitud que asuma el litigante a quien el fallo perjudica. Durante ese término existe un estado de suspensión de: los efectos.

Si no se introduce el recurso, la suspensión cesa y la sentencia pasa en cosa juzgada. Si el recurso se interpone, se crea un estado de cosas que luego será examinado. 
Pero, por el momento, mientras se halla pendiente el término para apelar, el acto jurídico se halla en suspenso.

Esa suspensión es a plazo y a condición. El plazo es el señalado en la ley; la condición, es la de que no se interponga el recurso.

Como todos los actos pendientes de condición suspensiva, si la condición no se cumple, el acto se tiene como puro y simples desde su fecha.

El problema de la retroactividad es, pues, en este caso, relativamente fácil de resolver. Si la sentencia fuere consentida, sus efectos se computan a partir de la fecha en que es notificado el fallo a las partes (9). Se produce así una retroactividad de los efectos propios de la sentencia ejecutoriada.

En el ejemplo arriba propuesto, si el bien inmueble fuere adquirido por el esposo durante los cinco días posteriores a la notificación del fallo y éste quedare ejecutoriado por ausencia de apelación, deve reputar-se que la sociedad conjugal quedó disuelta al notificarse la sentencia a las partes. El inmueble fué incorporado al patrimonio del adquirente fuera de la vigencia de la sociedad conyugal.

Distinta sería, en cambio, la situación que surgiría en lo que atañe a la retroactividad, si dictada una sentencia de divorcio ad vinculum de primera instancia, el cónyuge vencido muere durante el término para apelar. ¿Cabrá admitir,en ese caso, que el matrimonio ha sido disuelto?

No, en nuestro concepto. La acción de divorcio es personalísima y se sobresee por la muerte de uno de los cónyuges. Si uno de los esposos tenía aún derecho a

(8) C.c. 1421; "cum enim semel conditio extitit perinde habetur, ac si illo tempore quo stipulatio interposita est, sine conditione facta esset": ley 11,1,4, - L. 20, Digesto.

(9) No decimos hasta el día de su fecha puesto que hasta la notificación puede ser modificada por el juez: art. 486: C. P. C. Cfr. en este volúmen el estudio - Ampliación y rectificación de sentencia. 
interponer un recurso legal, y murió pendiente el plazo para ejercerlo, su situación es análoga a la que surge en el caso de muerte durante el curso de la instancia. La retroactividad es consecuencia del consentimiento del fallo apelado, vale decir de no haber realizado voluntariamente el acto de impugnación. Ese consentimiento no existe, ni puede presumirse, en caso de muerte.

\section{Naturaleza del fallo de segunda instancia, confirmatorio del de primera.}

Descartada la situación que surge cuando la sentencia de primera instancia queda ejecutoriada, examinemos 'la situación cuando ha sido interpuesto el recurso de apelación.

Interpuesto el recurso, el estado de suspensión continúa: appelatione pendente nihil innovandum.

Esta suspensión dura lo que dura la segunda instancia. Pero confirmado el fallo, se produce una homologación de la sentencia de primera instancia por acto del superior.

El fallo de primera instancia viene a quedar así como esos actos del derecho civil supeditados a confirmación. $\mathrm{Si}$ el acto es confirmado se han reunido los dos elementos: la voluntad originaria y la voluntad confirmatoria. Si no es confirmado, si ha fallado uno de los elementos, el acto no produce efectos.

La sentencia de primera instancia es una voluntad jurídica originaria que puede valer por sí sola si no se introduce el recurso de apelación; pero introducido el recurso, es una voluntad jurídica originaria sometida a confirmación por el superior. En consecuencia, sólo la voluntad originaria a la que se suma la voluntad confirmatoria, produce la cosa juzgada.

Estas ideas adquieren una particular significación no bien se examinan desde el punto de vista del contenido 
de la segunda instancia dentro de los processos de origen hispánico en América.

Como es sabido, las legislaciones europeas, particularmente la francesá, la alemana y la italiana, concibieron la segunda instancia como un verdadero proceso de revisión de todia la primera instancia. En el derecho alemán se llegó a hablar de "una segunda primera instancia" para significar este carácter de revisión plenaria del fallo de primera instancia. La legislación' española, por el contrario, nunca concibió una segunda instancia que fuera una repetición de la primera, sino que, por el contrario, limitó el contenido dela segunda instancia a una revisión del fallo de primera instancia. No se procuró dentro de ese sistema legal, reparar los errores cometidos en la primera instancia por las partes, sino tan sólo revisar el fallo para determinar su justicia o injusticia. Como es sabido, las reformas europeas de 1933 en Alemania, de 1935 en Francia y de 1940 en Italia, inclinaron aquellos sistemas al de la legislación española que nosotros hemos heredado (10).

Siendo así, siendo la segunda instancia un proceso de revisión de la sentencia de primera instancia, es necesario admitir que el fallo de primera instancia no queda completo hasta tanto no se ha cumplido la instancia de revisión, abierta por el recurso. El vencido tiene derecho a provocar la revisión del primer fallo por el superior y no puede decirse que el processo jurisdiccional quede cumplido hasta tanto esa etapa no se halle agotada.

Distinta sería la situación si, en lugar de tratarse de un recurso de apelación, se tratara de un recurso extraordinario de nulidad notoria o, en las legislaciones en que esto es posible, de un recurso autónomo de nulidad.

La sentencia que se pronuncia en esos casos, no confirma la sentencia impiugnada. Se limita a rechazar el

(10) Fundamentos del derecho procesal civil, p. 209. 
recurso o la impugnación de nulidad; y entonces el acto queda válido desde el día de su expedición. Todo sucedería en ese caso, como si la impugnación no se hubiere producido.

Pero tratándose de apelación, el acto jurídico que es la sentencia queda, en todos sus efectos, sometido a confir-mación; y sin la confirmación, no es virtualmente sentencia. Para decírlo con el lenguaje exacto de Chiovends, debe definirse como un acto jurídico que puede llegar a ser sentencia.

No parece admisible, frente a este sistema, la tésis de que la sentencia quede sometida a condición suspensiva o resolutoria. La función jurisdiccional se realiza en dos instancias ligadas entre sí por el principio de unidad de la relación procesal. El fallo de primera instancia es solamente una parte de esa unidad. La unidad completa la constituye la yuxtaposición de la primera y de la segunda instancia.

El fallo de primera instancia no tiene valor si no logra su aprobación por un tribunal. El fallo de segunda instancia no tiene significación si no tiene ante sí, como antecedente necesario, el fallo de primera instancia.

El mismo léxico de nuestros tribunales invita a pensar así. Nuestros jueces no acostumbran, en el fallo de segunda instancia, a decir: "Se rechaza el recurso de apelación declarándose infundado". Si así lo dijeran, podria pensarse que la sentencia ha sido mal apelada y que el recurso debe tenerse por no interpuesto dejándose las cosas tal como estaban el día del fallo de primera instancia. Por el contrario, nuestros fallos de segunda instancia dicen siempre, "se confirma" (o eventualmente, "se revoca") la sentencia apeladia. Esta terminología forense subraya el carácter constitutivo de la segunda instancia.

Resumiendo, pues, esta otra parte da nuestra exposición: la sentencia de segunda instancia confirmatoria de la de primera constituye, en sí misma, un acto de homo- 
logación o, como lo dice su propia denominación, de confirmación. Ella integra conjuntamente con el acto confirmado $u$ homologado, una unidad jurídica inseparable. No hay, en consecuencia, retroactividad, y los efectos nacen a partir del momento en que el proceso de la voluntad jurídica queda completo mediante la unión del acto confirmado y del acto confirmatorio

\section{Naturaleza del fallo de segunda instancia, revocatorio del de primera.}

¿Pero cuál es la situación si el fallo de segunda instancia revoca?

No podremos hablar, en ese caso, de un acto jurídico compuesto de dos etapas, una sujeta a confirmación y otra confirmatoria. Si el ordenamiento procesal admite, como en caso del derecho uruguayo, una tercera instancia en determinadas condiciones, entonces el segundo fallo renueva el planteo del primero y queda a su vez supeditado a confirmación. Pero si no existe tercera instancia, como ocurre en la mayoría de las leyes procesales vigentes y en muchas situaciones de nuestro propio derecho, entonces el fallo de segunda instancia hace cosa juzgada por sí solo, sea cual fuere el contenido del fallo de primera instancia.

No puede hablarse, entonces, de acto de confirmación, complementario del acto confirmado. Debe hablarse de acto autónomo, que produce sus efectos a pesar de su caráter revocatorio.

Esto es, efectivamente, así. Pero ese acto, aparentemente autónomo no quedaría explicado sino estuviera ligado al de primera instancia por el principio de unidad de la relación procesal. La sentencia de segunda instancia no produce efectos por sí sola; los produce en razón de constituir un pronunciamento sobre la sentencia de pri- 
mera instancia. Su carácter revocatorio es sólo una contingencia en la vida del proceso. Siempre se tratará de una sentencia de segunda instancia, surgida en razón del recurso interpuesto contra la de primera. Sin esta sentencia de primera instancia y sin el recurso de apelación, la sentencia de segunda instancia no tendíia explicación. alguna.

Convengamos, pues, en que la razón de ser del fallo: de segunda instancia es el fallo de primera instancia. No puede decirse que produce efectos con abstracción del fallo de primera. Por el contrario, confirmatorio o revocatorio, el fallo de segunda instancia constituye una unidad con el fallo de primera instancia. Los efectos del de segunda, acogiendo o rechazando la demanda, prevalecen sobre los efectos del de primera; pero no pueden prevalecer sin el fallo de primera, del cual son una continuación. necesaria y el cual es un antecedente también necesario.

En conclusión: el fallo de segunda instancia, ya sea confirmatorio o revocatorio del de primera, es siempre cons-titutivo de efectos jurídicos. Estos se logran en ambos. casos merced a la unidad que liga ambos fallos; si los dos son de idéntico sentido; el de segunda es un acto ho-mologatorio y de confirmación; y si son de diferente contenido, prevalece el de segunda, por imperativo de la ley; pero siempre en subordinación al fallo de primera instan-cia, sin el cual no puede existir el de segunda.

6. Irretroactividad de la sentencia de segunda instancia.

Siendo las cosas de este modo, y siendo el fallo de segunda instancia de carácter constitutivo, sus efectos se. producen para el futuro y no existe retroatividad de los. mismos hacia el pasado. 
En los actos jurídicos de carácter declarativo, el acto no altera la situación anterior: solamente la declara. Los efectos existen, pues, desde el momento en que se produjo el acto ulteriormente declarado. Por el contrario, en los actos de carácter constitutivo, aquellos que crean derechos -que no existían antes de su realización, los efectos se producen a partir del acto creativo (11).

La sentencia de segunda instancia no escapa a estas reglas; y habiendo admitido su carácter constitutivo, cabe concluir que sus efectos no tienen retroactividad a la fecha de la sentencia de primera instancia, produciendo sus efectos hacia el futuro.

Dictada la sentencia de divorcio de segunda instancia, no puede suponerse que sus efectos se retrotraen hacia la de primera instancia. Por el contrario, el matrimonio queda disuelto solamente a partir de la sentencia de segunda instancia.

Distinta seria la solución en los casos de sentencias de mera declaración o de condena. Pero esto no ocurre en razón de la naturaleza de la sentencia de segunda instancia, sino erı razón de la naturaleza del derecho que en el proceso se está debatiendo.

En efecto: según hemos tenido oportunidad de exponerlo en otro lugar (12), las sentencias de mera declaración tienen una retroactividad total. Cuando se declara judicialmente adquirida la propiedad por prescripción, la sentencia que declara adquirido el dominio retrotrae sus efectos al día en que, transcurridos los treinta años de posesión útil, se consumó la usucapión. La retroactividad es total y la sentencia se limita a declarar la existencia de un

(11) Fundamentos del derecho procesal civil, p. 184; asimismo, Declaración judicial de la prescripción adquisitiva en estos Estudios. II, p. 313.

(12) Fundamentos del derecho procesal civil, p. 181. 
derecho ya existente en el patrimonio del actor con anterioridad a la demanda (13).

Por su parte, las sentencias de condena retrotraen sus efectos al día de la demanda. Esto no acontece, en nuestro derecho, según hemos intentado demostrarlo en la anterior oportunidad aludida, por razón de justicia, sino por razón de especiales argumentos de derecho positivo que así lo establecen.

En cambio las sentencias constitutivas, tal como la de divorcio que venimos examinando, proyectan sus efectos hacia el futuro. El matrimonio subsiste hasta la sentencia ejecutoriada y el divorcio proyecta sus consecuencias para el futuro y no para el pasado.

\section{Conclusión}

Dentro de nuestro derecho positivo la solución expuesta adquiere particular consistencia, por virtud de algunos argumentos y razones que expondremos a título de conclusión.

Conviene colocar en un primer plano la disposición del art. 170 del Código Civil.

Dice esta disposición: "Comienzan entre los cónyuges los efectos de la separación desde el día en que pasare en autoridad de cosa juzgada la sentencia pronunciada en el juicio respectivo".

A estar a este texto es "el día en que pasare en autoridad de cosa juzgada la sentencia" el que determina los efectos jurídicos de la misma. Ese día no es sino el día de la sentencia de segunda instancia confirmatoria de la

(13) Cfr. Declaración judicial de la prescripción adquisitiva e Estudios de Derecho Procesal Civil". Buenos Aires 1939 T. II .p. 366 . 
primera o el día de la sentencia de tercera instancia. Los términos de la ley parecerían escritos con el propósito de no dejar equívocos sobre este asunto.

En segundo término habría que incluir, siempre dentro de nuestro derecho positivo, la disposición del art. 490 C. P. C.. Según dicha disposición las sentencias adquiren autoridad de cosa juzgada "cuando la ley no permite en el pleito, otra instancia o recurso ordinario". Quiere decir que los términos del texto legal parecerían conducir a la idea de la no retroactividad de la sentencia de segunda instancia. Si la cosa juzgada se opera desde el momento en que un fallo no admite recurso ulterior, la fecha de éste es la que fija el derecho. Cuando hay dos sentencias conformes, o disconformes, pero esta última no susceptible de recurso alguno, no es la fecha del fallo de primera instancia la que hace la cosa juzgada, sino la del segundo.

Siendo asil las cosas, hallámos frente a una sentencia doblemente constitutiva (constitutiva en cuanto sentencia de segunda instancia y constitutiva en cuanto sentencia de divorcio) los efectos se proyectan hacia el futuro y el inmueble adquirido durante la segunda instancia pertenece a la sociedad conyugal y no al cónyuge que lo adquirió a su nombrie.

Se dirá, acaso, que la solución es injusta si es que el marido ha adquirido el inmueble con el fruto de su trabajo posterior a la separación de hecho de ambos conyuges. Pero para contestar esta objeción, cabe advertir que ella se refiere a la justicia de nuestras leyes y no a la justicia de nuestras interpretaciones. Como es sabido, a diferencia del derecho argentino que atribuye efectos jurídicos a la separación de hecho de los cónyuges, nuestras leyes no le atribuyen efecto jurídico a la separación. La sociedad conyugal se disuelve solamente por muerte o por sentencia y los bienes adquiridos dentro de ella son nece- 
sariamente comunes, salvo el caso de subrugación o las nuevas soluciones aportadas por la ley 10.783 .

El cónyuge que adquirió el bien inmueble antes de la cosa juzgada sabía o debía saber que la ley atribuía a esos bienes un carácter ganancial. Y siendo así, los riesgos inherentes al acto de adquisisión han resultado asumidos por él.

Por otra parte, y para concluir con un argumento de hecho al que debemos atribuir importancia práctica, sabe subrayar que, salvo que se trate de un caso de adquisisión instantánea del dinero necesario para comprar el inmueble, lo que cabe presumir es que ese dinero se hallaba en el patrimonio de la sociedad conyugal al tiempo de la sentencia de primera instancia, dado que el curso de la segunda instancia no es muy prolongado. El cónyuge que tenia ese dinero a su disposición debía a su otro consorte la restitución de su cuota indivisa en ese dinero. La participacion en el inmueble, no es, pues, otra cosa que un sucedáneo de la participación en dinero necesario para adquirirlo.

Examinadas así las cosas, se comprende todavía que la solución es menos injusta de lo que parece a primera vista.

La doctrina parece también ponerse del lado de la justicia. 